\title{
A ESTRUTURA DE CAPITAL É RELEVANTE PARA A RENTABILIDADE DOS BANCOS? EVIDÊNCIAS EMPÍRICAS NOS MAIORES BANCOS BRASILEIROS COM PAPÉIS NEGOCIADOS NA [B] ${ }^{3}$
}

\author{
Alexandre Franco de Godoi ${ }^{1}$, José Odálio dos Santos, Fernando de Almeida Santos \& José Carlos Marion \\ Pontifícia Universidade Católica de São Paulo - PUC, São Paulo, SP (Brasil)
}

\section{DETALHES DO ARTIGO}

\section{Histórico do Artigo}

Recebido em: 14 de outubro de 2019

Aceito: 28 de setembro de 2020

Disponível online: 01 de janeiro de 2021

Sistema de revisão "Double blind review"

Editor Científico

Ilan Avrichir

\section{Palavras-chaves:}

Relevância da Estrutura de Capital. Determinantes da Rentabilidade Bancária.

Desempenho dos Bancos.

\section{RESUMO}

Objetivo: Identificar evidências da relevância da estrutura de capital e das principais estratégias bancárias para a maximização de valor nos bancos.

Método: Pesquisa de natureza empírico-analítica, descritiva, de abordagem quantitativa, sendo os dados longitudinais coletados da base de dados Economatica ${ }^{\circledR}$ entre o período do 10 trimestre de 2008 ao 4 으 trimestre de 2018. Para as análises foram realizados testes de regressão linear com dados em painel (panel data).

Principais resultados: Há evidências da relevância da estrutura de capital e da influência de estratégias bancárias para a rentabilidade dos bancos mensurada com base no ROIC AJUSTADO.

Relevância e originalidade: A atualidade, relevância e debates críticos inevitáveis sobre o tema desperta o interesse da área acadêmica por pesquisas direcionadas à análise crítica da relevância da estrutura de capital e de estratégias operacionais para a maximização de valor.

Contribuições teóricas: Oportuniza o debate acadêmico inevitável acerca da relevância da estrutura de capital e das principais estratégias bancárias para a maximização de valor nos maiores bancos de capital aberto.

Contribuições sociais: Contribui para o processo decisório de gestores, investidores, analistas financeiros e órgãos reguladores do setor ao abordar a relevância da estrutura de capital para os bancos, os riscos e custos envolvidos, os principais aspectos que incentivam níveis elevados de alavancagem financeira e os fatores determinantes para a rentabilidade.

\section{Introdução}

A administração da estrutura de capital nos bancos envolve decisões que devem ser tomadas de maneira eficiente quando da captação de depósitos e administração das necessidades de reservas. Uma vez respeitadas as restrições impostas pela regulamentação do setor financeiro, os bancos fazem uso do capital de terceiros e do capital próprio como fonte de recursos para o financiamento de novos projetos e expansão das operações (Saunders, 2000). Evidentemente, há uma preferência pelos bancos em operar com recursos de terceiros na estrutura de capital, principalmente maiores níveis de depósitos de clientes (Assaf, 2015; Mendonça, Souza, Benedicto, \& Silva, 2016).

Essa forma de captação de recurso é considerada menos onerosa pelo fato de ser obtida a taxas de juros reduzidas, geralmente, abaixo da taxa livre de risco da economia, ou até mesmo, sem nenhum ônus, como ocorre com os depósitos à vista, criando assim as condições ideais para um possível incremento da rentabilidade bancária (Vieira, 2016). Em razão disso, a participação do capital próprio na estrutura de capital dessas empresas acaba se tornando bastante reduzida, geralmente, em média, $11 \%$ do capital emprestado em atendimento às exigências de capital

\footnotetext{
${ }^{1}$ Contato do autor - E-mail: godoi franco@outlook.com
} 
regulatório previsto nos Acordos de Basileia (Assaf, 2015).

Como os bancos atuam dentro de mercados reais e imperfeitos, determinados aspectos parecem distanciar as circunstâncias reais do ambiente em que atuam essas empresas da abordagem da Irrelevância da Estrutura de Capital (Octavia \& Brown, 2008). Dentre esses aspectos podem ser mencionados: o benefício fiscal pelo uso de dívidas na estrutura de capital (Orgler \& Taggart, 1983); o risco de insolvência e os custos privados de falência (Saunders, 2000; Jucá, 2011); custos de agência originados do conflito de interesses entre administradores e acionistas (Jensen, 1986), credores e acionistas (Jucá, 2011), e o uso do endividamento para disciplinar a atuação dos administradores (Jensen, 1986); o contexto da assimetria de informações (Aboura \& Lépinette, 2015) que torna mais oneroso os custos de emissão do capital próprio (Wall \& Peterson, 1998); as garantias e proteções governamentais e privadas sob a forma de seguros de depósitos, garantias implícitas de resgate e operações de redesconto (Aboura \& Lépinette, 2015; Jucá, 2011); e os requerimentos de capital regulatório previstos nos Acordos de Basileia (Octavia \& Brown, 2008; Belém \& Gartner, 2016).

Essas imperfeições existentes nos mercados, ao mesmo tempo em que podem incentivar os bancos a elevar os níveis de alavancagem financeira na estrutura de capital, fazem com que essas empresas tenham que privilegiar certos níveis de capital próprio na estrutura de capital que seja compatível com as expectativas de mercado, principalmente em razão dos custos e riscos a que ficam expostas na hipótese de maiores níveis de endividamento na estrutura de capital (Berger, Herring, \& Szegö, 1995; Jokipii \& Milne, 2008) e das penalidades aplicáveis por órgãos reguladores do setor no caso de não observância das exigências de capital regulatório (Rime, 2001). Posto isto, poder-se-ia admitir, ainda que de modo intuitivo inicialmente, a relevância da estrutura de capital para os bancos (Aboura \& Lépinette, 2015).

Como os bancos convivem com diversas restrições e controles legais em seus passivos, ainda persistem discussões quanto à capacidade dessas empresas criarem valor a partir de seus passivos (Assaf, 2015). Em outras palavras, não há ainda um consenso definitivo a respeito da irrelevância ou relevância da estrutura de capital para criação de valor nos bancos. Logo, esta pesquisa procura responder, por meio de uma abordagem quantitativa, ao seguinte problema de pesquisa: A estrutura de capital é relevante para a rentabilidade dos quatro maiores bancos de capital aberto no Brasil?

O objetivo geral da pesquisa é verificar a relevância da estrutura de capital para a rentabilidade dos maiores bancos de capital aberto no país. Como objetivos específicos buscam-se identificar evidências que sustentem a relevância da estrutura de capital para os bancos e verificar se as principais estratégias bancárias relacionadas à estrutura de capital, eficiência operacional, solvência e liquidez seriam relevantes para a maximização de valor. Para se investigar o problema de pesquisa, são propostas as seguintes hipóteses de pesquisa:

$H_{0}$ : Não há relação estatisticamente significativa entre a estrutura de capital e a rentabilidade dos bancos.

$\mathrm{H}_{1}$ : Existe relação estatisticamente significativa entre a estrutura de capital e a rentabilidade dos bancos.

$\mathrm{H}_{1.1}$ : A alavancagem financeira é relevante para a maximização de valor, admitindo-se que ela influencia positivamente a rentabilidade dos bancos.

$\mathrm{H}_{1.2}$ : A capitalização dos bancos é relevante para a maximização de valor e admite-se que ela influencia de forma positiva a rentabilidade dos bancos.

$\mathrm{H}_{1.3}$ : As estratégias bancárias relacionadas à eficiência operacional, solvência e liquidez também influenciam a rentabilidade dos bancos.

Um conjunto com proxies representativas dos principais fatores capazes de afetar a rentabilidade dos bancos, calculado por meio de indicadores econômico-financeiros, foi extraído das demonstrações financeiras para se testar as hipóteses de pesquisa. Optou-se pelo emprego de indicadores a valor contábil, dado a dificuldade de se obter informação a valor de mercado para parte dos indicadores utilizados nas análises (Coelho, 2015).

A pesquisa contribui no campo teórico ao oportunizar o debate crítico inevitável acerca da relevância da estrutura de capital e das principais estratégias bancárias para a maximização de valor nos maiores bancos com ações negociadas na $[B]^{3}$ Brasil, Bolsa, Balcão, assunto esse que desperta o interesse de agentes tomadores de decisões, órgãos reguladores e da própria comunidade acadêmica. No campo social, espera-se que ela possa contribuir para o processo decisório de gestores, investidores, 
analistas financeiros e órgãos reguladores do setor ao abordar a relevância da estrutura de capital para os bancos, os riscos e custos envolvidos, os principais aspectos que incentivam essas empresas a manterem níveis elevados de alavancagem financeira na estrutura de capital e os principais fatores determinantes para a sua rentabilidade.

\section{Desenvolvimento}

\section{Principais aspectos da atividade dos bancos e de sua estrutura de capital}

Os bancos apresentam características exclusivas que os diferenciam das empresas não financeiras. Como entidades públicas ou privadas, com finalidades lucrativas, os bancos como depositários da poupança de famílias, empresas e investidores institucionais, atuam como intermediários financeiros entre investidores e tomadores de recursos (Pinheiro, Savóia, \& Securato, 2015), desempenhando papel determinante para o funcionamento dos sistemas econômicos e desenvolvimento dos países (Belém \& Gartner, 2016).

Ao promover a realização de operações de empréstimos de recursos advindos, em grande parte, dos seus depositantes, os bancos viabilizam o fluxo de oferta de crédito na economia (Diamond \& Rajan, 2001) e determinam a criação do poder de compra necessário para empresas e pessoas físicas (Maffili, Bressan, \& Souza, 2007), sendo provedores de liquidez e segurança ao sistema financeiro e indutores do desenvolvimento pela concessão de crédito para consumo e investimentos (Andrade, 2014).

A complexidade e diversidade da atividade bancária expõem os bancos a vários tipos de riscos que podem fragilizar a sua situação financeira (Belém \& Gartner, 2016). Para garantir um ambiente seguro e de solidez para o sistema financeiro, evitando-se assim que perdas inesperadas ou problemas financeiros a que estejam submetidos os bancos possam comprometer como um todo o sistema de intermediação financeira (Vallascas \& Hagendorff, 2013) e produzir efeitos prejudiciais para a economia dos países como mostram as crises sistêmicas (Saunders, 2000), órgãos reguladores do setor tem exigido dos bancos a manutenção de capital regulatório em níveis suficientes para suportar os riscos a que estão expostos os seus ativos (Belém \& Gartner, 2016).
Os recursos captados pelos bancos na estrutura de capital para o financiamento de novos projetos e expansão das operações são obtidos por meio do capital de terceiros e do capital próprio (Saunders, 2000). Há um elevado nível de endividamento na estrutura de capital dos bancos, dada a preferência em operar com recursos do capital de terceiros, tornando assim bastante reduzida a participação do capital próprio que é utilizado para definir os limites de empréstimos e demais operações ativas, mitigar a exposição aos riscos e cobrir eventuais perdas (Assaf, 2015).

Maiores níveis de depósitos compondo a estrutura de capital dos bancos geram um maior volume de recursos disponíveis para a intermediação financeira (Mendonça et al., 2016) e representam uma forma de captação menos onerosa para incremento da lucratividade bancária, dadas as garantias que são oferecidas por órgãos privados ou governamentais para redução dos riscos e dos custos de captação (Vieira, 2016), essas geralmente sob a forma de seguros de depósitos ou outras garantias implícitas de resgate (Jucá, 2011).

Pode-se afirmar que os bancos não se enquadram no tipo de empresas clássicas não financeiras que assume a proposição de Irrelevância da Estrutura de Capital (Modigliani \& Miller, 1958), uma vez que as garantias e proteções oferecidas por governos e órgãos privados reduzem os níveis de riscos no setor bancário e criam estímulos para elevados níveis de alavancagem financeira na estrutura de capital (Aboura \& Lépinette, 2015). A presença de certas imperfeições existentes nos mercados é outro aspecto que pode afetar a estrutura de capital dos bancos, o que permite intuir pela sua relevância para a maximização de valor (Aboura \& Lépinette, 2015), distanciando assim as circunstâncias reais do ambiente em que operam os bancos da abordagem da Irrelevância da Estrutura de Capital (Octavia \& Brown, 2008).

A composição da estrutura de capital nos bancos pode ser influenciada e explicada por fatores relacionados a atributos das empresas bancárias, efeitos macroeconômicos e a regulação existente no setor bancário (Romdhane, 2010). E sua rentabilidade, determinada por fatores como margem de lucro, eficiência operacional, planejamento eficiente e a elevada dependência de recursos de terceiros (leverage) na estrutura de 
capital, aspecto esse que possibilita aos bancos a alavancagem de seus resultados (Assaf, 2015).

Pesquisas empíricas têm sido desenvolvidas com o objetivo de analisar a influência que fatores operacionais da atividade bancária, em especial as decisões de estrutura de capital, aspectos macroeconômicos, e exigências regulatórias inerentes ao setor, exercem sobre o desempenho dos bancos. A Tabela 1 relaciona algumas pesquisas realizadas no Brasil e seus principais resultados.

\section{Tabela 1}

Evidências empíricas da análise do desempenho dos bancos no Brasil

\begin{tabular}{|c|c|c|}
\hline Autores & Período & Principais resultados \\
\hline Bittencourt et al. (2017) & $2009-2013$ & $\begin{array}{l}\text { O ROA é afetado por empréstimos, eficiência, despesas totais, depósitos } \\
\text { totais, outras receitas e Selic. O ROE é influenciado por depósitos totais, } \\
\text { empréstimos, Selic, PIB, inflação, outras receitas e despesas totais. }\end{array}$ \\
\hline $\begin{array}{l}\text { Caliman, Sarlo Neto, } \\
\text { Reina e Junior (2016) }\end{array}$ & $2012-2015$ & $\begin{array}{l}\text { Margem Líquida e ROA têm relação positiva com ROE. Endividamento, } \\
\text { Independência Financeira e Eficiência Operacional têm relação negativa. O } \\
\text { Índice de Basileia não apresentou significância estatística. }\end{array}$ \\
\hline Mendonça et al. (2016) & 2011-2015 & $\begin{array}{l}\text { Relação estatisticamente significativa entre eficiência econômico-financeira } \\
\text { e lucratividade. Quanto maior a eficiência econômico-financeira do banco, } \\
\text { maior a lucratividade obtida. }\end{array}$ \\
\hline Vieira (2016) & 1996-2015 & $\begin{array}{l}\text { Bancos mais endividados possuem menor ROA e maior ROE. A captação de } \\
\text { depósitos aumenta a lucratividade dos bancos, enquanto que maior } \\
\text { diversificação das operações ocasiona menor retorno. }\end{array}$ \\
\hline $\begin{array}{l}\text { Mantovani e Santos } \\
(2015)\end{array}$ & $2001-2010$ & $\begin{array}{l}\text { Relação significativa entre indicadores de alavancagem financeira bancária e } \\
\text { indicadores de rentabilidade. }\end{array}$ \\
\hline D’Oliveira (2014) & 1995-2013 & $\begin{array}{l}\text { Tamanho do banco, financiamento, capitalização, liquidez, risco de crédito, } \\
\text { crescimento de crédito, eficiência técnica, concentração e controle de capital } \\
\text { pelo governo e taxa de juros foram estatisticamente significativas para a } \\
\text { rentabilidade dos bancos. }\end{array}$ \\
\hline $\begin{array}{l}\text { Primo, Dantas, Medeiros } \\
\text { e Capelletto (2013) }\end{array}$ & 2000-2009 & $\begin{array}{l}\text { Taxa básica de juros, PIB, carga tributária, eficiência operacional e } \\
\text { participação dos bancos nacionais têm relação positiva com a rentabilidade. } \\
\text { Variação Cambial tem relação negativa e o Nível de Inflação tem sinais } \\
\text { distintos dependendo do modelo aplicado. }\end{array}$ \\
\hline $\begin{array}{l}\text { Gonçalves, } \quad \text { Tavares, } \\
\text { Ximenes e Silva (2012) }\end{array}$ & 2008-2009 & $\begin{array}{l}\text { Os bancos tendem a adotar posturas conservadoras e tendências anticíclicas } \\
\text { ao fomentar as atividades produtivas, com preferência por maior liquidez em } \\
\text { prejuízo da rentabilidade. }\end{array}$ \\
\hline $\begin{array}{l}\text { Rover, Tomazzia e } \\
\text { Fávero (2011) }\end{array}$ & $1995-2009$ & $\begin{array}{l}\text { Liquidez, risco de crédito, despesas operacionais, eficiência operacional, } \\
\text { alavancagem, inflação e taxa Selic são estatisticamente significativas para a } \\
\text { rentabilidade bancária. }\end{array}$ \\
\hline $\begin{array}{l}\text { Oliveira, Macedo e } \\
\text { Corrar (2011) }\end{array}$ & $2008-2009$ & $\begin{array}{l}\text { Eficiência Operacional e Retorno das Operações de Crédito são aspectos } \\
\text { problemáticos na avaliação do desempenho. Mudanças nas participações } \\
\text { acionárias dos bancos serviram para manter ou melhorar os índices de } \\
\text { desempenho. }\end{array}$ \\
\hline $\begin{array}{l}\text { Martin, Kimura, Kayo e } \\
\text { Santos (2011) }\end{array}$ & $1996-2010$ & $\begin{array}{l}\text { Identificou-se uma relação positiva e estatisticamente significativa da } \\
\text { diversificação, qualidade da gestão e eficiência tecnológica com a } \\
\text { lucratividade bancária. A relação negativa entre capacidade de investimento } \\
\text { e lucratividade foi estatisticamente significativa. }\end{array}$ \\
\hline Souza e Macedo (2009) & 2001-2005 & $\begin{array}{l}\text { A eficiência econômico-financeira dos bancos está relacionada ao baixo custo } \\
\text { operacional e alta rentabilidade. }\end{array}$ \\
\hline $\begin{array}{l}\text { Maffili, Bressan e Souza } \\
\text { (2007) }\end{array}$ & $1999-2005$ & $\begin{array}{l}\text { Operações de tesouraria, operações de crédito, estrutura de capital, índice } \\
\text { de eficiência e spread bancário têm relação estatística significativa com a } \\
\text { rentabilidade dos bancos de varejo. }\end{array}$ \\
\hline $\begin{array}{l}\text { Ceretta e Niederauer } \\
(2001)\end{array}$ & 1999 & $\begin{array}{l}\text { Com base no enfoque integrado entre eficiência operacional e rentabilidade } \\
\text { pela matriz BCG, bancos de grande porte apresentam o melhor desempenho, } \\
\text { enquanto os de pequeno porte o pior. }\end{array}$ \\
\hline
\end{tabular}

Fonte: Elaborado pelos autores 
Os bancos comportam-se como firmas que buscam a otimização racional de lucros (Vinhado \& Divino, 2013). Em outras palavras, eles têm por objetivo a maximização da rentabilidade, essa uma variável de natureza fundamental para a estabilidade do sistema bancário (D’Oliveira, 2014). Assim sendo, para que a relevância da estrutura de capital para a maximização de valor nos bancos possa ser comprovada, ela necessita ser objeto de pesquisa empírica e analisada com o devido rigor metodológico e adequado tratamento estatístico.

\section{Métodos de pesquisa}

A pesquisa é de natureza empírico-analítica, por utilizar técnicas de coleta, tratamento e análise de dados quantitativos, com forte preocupação em estabelecer a relação causal entre variáveis (Martins, 2002). Sua abordagem é quantitativa, sendo um meio para testar teorias objetivas e examinar a relação entre variáveis mediante o emprego de procedimentos estatísticos (Creswell, 2010). Quanto aos objetivos, trata-se de uma pesquisa descritiva, pois proporciona como resultado um quadro de determinado fenômeno (Gray, 2012).

Os dados coletados são longitudinais e originam-se de fontes secundárias. Para cálculo das variáveis de natureza econômico-financeiras utilizou-se as demonstrações financeiras trimestrais consolidadas disponíveis na base de dados Economatica ${ }^{\circledR}$, tendo sido os dados trimestrais da Demonstração do Resultado do Exercício (DRE) obtidos em bases anualizadas. A pesquisa contemplou o período de 2008 até 2018, o qual pode ser considerado apropriado para a análise, ainda que seja ele composto por períodos de baixa e alta volatilidade no mercado, num misto de momentos de longa estabilidade econômica interrompidos por fortes crises políticoeconômicas nacionais e internacionais.

O método de amostragem na pesquisa é classificado como não probabilístico por conveniência (Hair, Babin, Money, \& Samouel, 2005), pois na seleção dos bancos para a amostra optou-se por aqueles com significativa participação no sistema financeiro nacional e informações econômico-financeiras disponíveis para o desenvolvimento do estudo. Assim, a amostra da pesquisa é composta pelos 4 (quatro) maiores bancos com ações negociadas na [B] $]^{3}$ Brasil, Bolsa, Balcão. Dos bancos comerciais, bancos múltiplos com carteira comercial e caixas econômicas autorizados pelo Banco Central do Brasil (2019) a operar no país, somente os quatro maiores bancos de capital aberto - Banco do Brasil, Itaú Unibanco, Bradesco e Santander - concentraram em conjunto, ao final do ano de 2018, 64,30\% dos ativos totais; $60,77 \%$ das operações de crédito; 64,62\% da captação de depósitos; e 69,42\% do patrimônio líquido total, o que indica a representatividade dessas instituições financeiras para o setor bancário dentro do sistema financeiro nacional.

A variável dependente de pesquisa está relacionada à maximização de valor e ao desempenho dos bancos, sendo representada pela proxy de rentabilidade bancária Return on Invested Capital Adjusted (ROICAJustado). Já as variáveis independentes se referem às principais estratégias adotadas pelos bancos para maximização da rentabilidade. Elas são representadas por proxies relacionadas à estrutura de capital, risco de crédito, eficiência operacional, solvência e liquidez, e foram determinadas a partir de revisões realizadas em estudos empíricos sobre a rentabilidade bancária. A Tabela 2 apresenta as proxies representativas das variáveis da pesquisa e a relação esperada entre elas. 
Tabela 2

Variável Dependente e Variáveis Independentes da Pesquisa

\begin{tabular}{|c|c|c|}
\hline \multicolumn{3}{|c|}{ Variável Dependente da Pesquisa } \\
\hline $\begin{array}{l}\text { Retorno sobre o Capital Investido Ajustado } \\
\text { Adaptado de Chen (2014) }\end{array}$ & \multicolumn{2}{|l|}{ ROIC $_{\text {Ajustado }}=\frac{\text { NOPAT }_{t}}{\text { Capital Total Investido Ajustado }}$} \\
\hline \multicolumn{3}{|c|}{ Variáveis Independentes da Pesquisa e a relação esperada com a rentabilidade } \\
\hline $\begin{array}{l}\text { Capitalização } \\
\text { D'Oliveira (2014) }\end{array}$ & CAP $=\frac{\text { Patrimônio Líquido }}{\text { Ativo Total }}$ & $+/-$ \\
\hline $\begin{array}{l}\text { Leverage ou Multiplicador do P.L. } \\
\text { Assaf (2015) e Ross, Westerfield, Jaffe e Lamb (2015) }\end{array}$ & $L E V=\frac{\text { Ativo Total }}{\text { Patrimônio Líquido }}$ & $+/-$ \\
\hline $\begin{array}{l}\text { Custo Operacional } \\
\text { Mendonça et al. (2016) }\end{array}$ & $\begin{array}{l}\text { CUS } \\
=\frac{\text { Despesas com Pessoal }+ \text { ADM }+ \text { Interm.Fin. }}{\text { Receitas de Serviços }+ \text { Receitas Interm.Fin. }}\end{array}$ & - \\
\hline $\begin{array}{l}\text { Spread Bancário } \\
\text { Rover, Tomazzia e Fávero (2011) }\end{array}$ & $\begin{array}{l}S P R \\
=\frac{\text { Receitas Oper.Crédito }- \text { Despesas Captação }}{\text { Receitas Oper.Crédito }}\end{array}$ & + \\
\hline $\begin{array}{l}\text { Operações de Crédito } \\
\text { Gonçalves et al. (2012) }\end{array}$ & $C R E=\frac{\text { Volume Oper. Crédito e Arrend. Mercantil }}{\text { Ativo Total }}$ & + \\
\hline $\begin{array}{l}\text { Encaixe Voluntário } \\
\text { Gonçalves et al. (2012) }\end{array}$ & $E N C=\frac{\text { Disponibilidades }}{\text { Depósitos à Vista }}$ & - \\
\hline $\begin{array}{l}\text { Liquidez Imediata } \\
\text { Assaf (2015) }\end{array}$ & $\begin{array}{l}\text { LIQ } \\
=\frac{\text { Disponibilidades }+ \text { Aplic. Financ. de Liquidez }}{\text { Depósitos à Vista }}\end{array}$ & - \\
\hline $\begin{array}{l}\text { Risco de Crédito } \\
\text { D'Oliveira (2014) }\end{array}$ & $R I S=\frac{\text { Provisão Créditos Liquidação Duvidosa }}{\text { Operações de Créditos Totais }}$ & $+/-$ \\
\hline $\begin{array}{l}\text { Operações de Tesouraria } \\
\text { Maffili, Bressan e Souza (2007) }\end{array}$ & $T E S=\frac{\text { Aplicações Interfin. }+T V M+\text { Derivativos }}{\text { Ativo Total }}$ & + \\
\hline $\begin{array}{l}\text { Grau de Imobilização } \\
\text { Mendonça et al. (2016) }\end{array}$ & $I M O=\frac{\text { Ativo Imobilizado }}{\text { Patrimônio Líquido }}$ & - \\
\hline
\end{tabular}

Fonte: Elaborado pelos autores

Normalmente, as pesquisas empíricas têm se utilizado do Return on Assets (ROA) e do Return on Equity (ROE) como proxies para representar a rentabilidade dos bancos. O Return on Invested Capital (ROIC) possibilita uma melhor compreensão do desempenho operacional dos bancos ao ser comparado com métricas de retorno como o ROA e o ROE (Copeland, Koller, \& Murrin, 2002), enfatizando o entendimento de como essas empresas estão criando (ou destruindo) valor. O uso do Return on Invested Capital Adjusted (ROIC AJUSTADO$_{\text {), }}$ como a variável dependente da pesquisa, traz como diferencial ao Return on Invested Capital (ROIC), tradicionalmente utilizado pelo mercado, o ajuste ao cálculo do Capital Total Investido mediante a inclusão dos saldos de depósitos de poupança.

Pode-se entender que esses recursos apresentam características de longo prazo, dado o prazo médio efetivo de permanência desse tipo de depósito ser superior ao período de um ano. Ainda que contabilmente registrados no Passivo Circulante, em conformidade com o COSIF - Plano Contábil das Instituições do Sistema Financeiro Nacional do Banco Central do Brasil (2017), os depósitos de poupança, geralmente, apresentam prazo de vencimento indeterminado, liquidez diária e mantêm uma estabilidade de saldos ao longo dos anos, consubstanciando-se em uma fonte de financiamento que contribui com o resultado de longo prazo dos bancos por meio das operações de crédito imobiliário.

Pelo exposto, é possível aceitar uma metodologia alternativa para o cálculo do ROIC desde que o avaliador padronize um modelo de cálculo que possibilite comparações e apresente uma clara ideia do Capital Total Investido (Chen, 2014). A Tabela 3 apresenta os principais elementos e ajustes para o cálculo do Lucro Operacional após o Imposto de Renda (NOPAT) e do Capital Total Investido Ajustado utilizados na determinação do ROIC AJustado. $_{\text {. }}$ 
A Estrutura de Capital é Relevante para a Rentabilidade dos Bancos? Evidências Empíricas nos Maiores Bancos Brasileiros com Papéis Negociados na $[B] 3$

\section{Tabela 3}

Cálculo do NOPAT e do Capital Total Investido Ajustado

\begin{tabular}{l|l}
\hline Cálculo do NOPAT & Cálculo do Capital Total Investido Ajustado \\
\hline + Receitas de Intermediação Financeira & + Passivo Circulante \\
\hline (-) Despesas de Intermediação Financeira & (Depósitos de Poupança) \\
\hline = Resultado Bruto da Intermediação Financeira & + Passivo Exigível à Longo Prazo \\
\hline + ou (-) Outras Receitas/Despesas Operacionais & (Depósitos e demais Passivos de Longo Prazo) \\
\hline$=$ Resultado Operacional & + Patrimônio Líquido \\
\hline (-) Imposto de Renda e Contribuição Social sobre o Lucro & + Participação de Não Controladores \\
\hline = Resultado Operacional após o Imposto de Renda (NOPAT) & $=$ Capital Total Investido Ajustado \\
\hline
\end{tabular}

Fonte: Elaborado pelos autores

Logo, definidas a variável dependente e as variáveis independentes, a equação prevista com

as proxies representativas das variáveis da pesquisa pode ser assim descrita:

$$
\begin{aligned}
R O I C_{i t}=\beta_{0}+ & \beta_{1} C A P_{i t}+\beta_{2} L E V_{i t}-\beta_{3} C U S_{i t}+\beta_{4} S P R_{i t}+\beta_{5} C R E_{i t}-\beta_{6} E N C_{i t}-\beta_{7} L I Q_{i t} \\
& +\beta_{8} R I S_{i t}+\beta_{9} T E S_{i t}-\beta_{10} I M O_{i t}+\mu_{i t}
\end{aligned}
$$

Onde: ROICAJUSTADO (Retorno sobre o Capital Investido Ajustado); CAP (Capitalização); LEV (Leverage); CUS (Custo Operacional); SPR (Spread Bancário); CRE (Operações de Crédito); ENC (Encaixe Voluntário); LIQ (Liquidez Imediata); RIS (Risco de Crédito); TES (Operações de Tesouraria); e IMO (Grau de Imobilização).

Mediante o emprego de testes estatísticos paramétricos busca-se investigar a significância de determinadas proxies representativas de estratégias bancárias que estão relacionadas à estrutura de capital - em especial a sua relevância para a maximização de valor -, eficiência operacional, solvência e liquidez para a rentabilidade dos 4 (quatro) maiores bancos com ações negociadas na [B] ${ }^{3}$ Brasil, Bolsa, Balcão. Para comprovar ou refutar as hipóteses de pesquisa foi utilizado um modelo econométrico estimado pela técnica de regressão com dados em painel por meio do uso do software EViews ${ }^{\circledR} 10$.

A análise de regressão com dados em painel permite a obtenção de estimadores mais precisos para os modelos econométricos e estatísticas de testes mais poderosas (Wooldridge, 2002). Proporciona ainda maior confiabilidade e robustez aos resultados obtidos (Menezes, 2017), sendo de ampla utilização em estudos econométricos e nas ciências sociais aplicadas (Duarte, Lamounier, \& Takamatsu, 2007). São três as principais abordagens para os modelos de regressão com dados em painel, sendo: (i) dados empilhados (pooled); (ii) efeitos fixos (fixed effects); e (iii) efeitos aleatórios (random effects) (Greene, 2012). Alguns testes estão previstos para definição do modelo econométrico considerado mais adequado e consistente nesse tipo de análise, sendo o Teste $F$ de Chow, o Teste de Hausman e o Teste do Multiplicador de Lagrange (LM) de Breusch e Pagan (Gujarati \& Porter, 2011).

Para cada banco que compõe a amostra de pesquisa há um número $T$ de observações ao longo do tempo. Assim, o número total de observações na pesquisa corresponde a $n \times T$, ou seja, configurando-se na formação de um conjunto de dados de corte transversal com 4 bancos associados a uma série temporal de 44 trimestres, totalizando 176 observações. Logo, trata-se de um painel longo, quando o número de períodos de tempo $T$ é maior que o número de sujeitos de corte transversal $n$, e do tipo balanceado pelo fato de cada unidade de corte transversal ter o mesmo número de observações.

\section{Resultados}

Inicialmente, para confirmar se a variável dependente da pesquisa apresenta uma distribuição normal, a pesquisa utilizou-se do Teste de Normalidade Jarque-Bera. O resultado obtido para o p-value > 0,05 (0,679529) da variável dependente (ROIC AJUSTADO$_{\text {) }}$ leva à aceitação da hipótese nula $\left(\mathrm{H}_{0}\right)$ de normalidade. Em outras 
palavras, os dados para a variável dependente da pesquisa são distribuídos normalmente. A Tabela 4 apresenta os resultados obtidos com as estatísticas do Teste de Normalidade Jarque-Bera.

\section{Tabela 4}

Estatísticas do Teste de Normalidade Jarque-Bera

\begin{tabular}{l|r}
\multicolumn{1}{c|}{ Teste de Normalidade } & \multicolumn{1}{c}{ Variável Dependente da Pesquisa } \\
\cline { 2 - 2 } & ROIC \\
\hline № de observações & 176 \\
\hline Jarque-Bera & 0,772711 \\
\hline Probability (p-value) & 0,679529 \\
\hline
\end{tabular}

Hipóteses para o Teste Jarque-Bera ( $\mathrm{p}$-value $<0,05$ rejeita-se a hipótese nula $\mathrm{H}_{0}$ )

$\mathrm{H}_{0}$ : Os dados da pesquisa são distribuídos normalmente

$\mathrm{H}_{1}$ : Os dados da pesquisa não são distribuídos normalmente

Fonte: Dados da pesquisa (2019).

As estimações para os testes de regressão com dados em painel foram realizadas no Modelo Agrupado (Pooled), Modelo com Efeitos Aleatórios (Random Effects) e Modelo com Efeitos Fixos (Fixed Effects). Para definição do modelo econométrico considerado mais adequado e consistente foram utilizados o Teste do Multiplicador de Lagrange (LM) de Breusch e Pagan (Teste Breusch-Pagan LM), o Teste F de Chow e o Teste de Hausman.

$\mathrm{Na}$ análise de regressão com dados em painel, foram inicialmente estimados o Modelo Agrupado (Pooled) e o Modelo com Efeitos Aleatórios (Random Effects). O Teste Breusch-Pagan LM foi aplicado para comparar entre o Modelo Agrupado (Pooled) e o Modelo com Efeitos Aleatórios (Random Effects). A significância estatística apresentada pelo $p$-value $<0,05(0,0000)$ levou à rejeição da hipótese nula que prevê haver adequação do Modelo Agrupado (Pooled) em relação ao Modelo com Efeitos Aleatórios (Random Effects). Por conseguinte, foi então aceita a hipótese alternativa que considera o Modelo com Efeitos Aleatórios (Random Effects) como o mais adequado e consistente para as estimativas de regressão com dados em painel para os dados da pesquisa. Os resultados para o Teste BreuschPagan LM podem ser verificados na Tabela 5.

\section{Tabela 5}

Teste Breusch-Pagan LM - Regressão Variável Dependente ROIC AJUSTADO

\begin{tabular}{l|c|c|c}
\hline Teste & Estatística & Graus de Liberdade & Probabilidade \\
\hline Breusch-Pagan LM & 61,54529 & 6 & 0,0000 \\
\hline Hipóteses para o Teste Breusch-Pagan LM (p-value < 0,05 rejeita-se a hipótese nula Ho) & \\
\hline Ho: Há adequação do Modelo Pooled em relação ao Modelo com Efeitos Aleatórios. (Modelo Pooled) \\
\hline Hón : Não há adequação do Modelo Pooled em relação ao Modelo com Efeitos Aleatórios. (Efeitos Aleatórios) \\
\hline
\end{tabular}

Em seguida, foi realizada a estimação do teste de regressão com dados em painel no Modelo com Efeitos Fixos (Fixed Effects). O Teste F de Chow foi aplicado para examinar a estabilidade estrutural do modelo de regressão e verificar a melhor alternativa entre o Modelo Agrupado (Pooled) e o Modelo com Efeitos Fixos (Fixed Effects). A significância estatística apresentada pelo $p$-value < $0,05(0,0000)$ levou a rejeição da hipótese nula que declara haver igualdade de interceptos e inclinações para toda a amostra em análise (modelo pooled o preferível). Logo, é aceita a hipótese alternativa que considera o Modelo com Efeitos Fixos (Fixed Effects) como o mais adequado para os dados da pesquisa. Os resultados obtidos com o Teste F de Chow são apresentados na Tabela 6. 
A Estrutura de Capital é Relevante para a Rentabilidade dos Bancos? Evidências Empíricas nos Maiores Bancos Brasileiros com Papéis Negociados na $[B] 3$

\section{Tabela 6}

Teste de Chow - Regressão com a Variável Dependente ROICAJustado

\begin{tabular}{l|r|r|r}
\hline Teste & \multicolumn{1}{|c|}{ Estatística } & \multicolumn{1}{l|}{ Graus de Liberdade } & \multicolumn{1}{l}{ Probabilidade } \\
\hline Cross-section F & 38,950128 & $(3,119)$ & 0,0000 \\
\hline Cross-section Chi-quadrado & 120,397049 & 3 & 0,0000 \\
\hline Período F & 4,031670 & $(43,119)$ & 0,0000 \\
\hline Período Chi-quadrado & 158,200866 & 43 & 0,0000 \\
\hline Cross-Section/Período F & 8,071288 & $(46,119)$ & 0,0000 \\
\hline Cross-Section/Período Chi-quadrado & 249,189894 & 46 & 0,0000 \\
\hline
\end{tabular}

Hipóteses para o Teste de Chow ( $p$-value $<0,05$ rejeita-se a hipótese nula $\mathrm{H}_{0}$ )

$\mathrm{H}_{0}$ : Há igualdade de interceptos e inclinações para toda a amostra. (Modelo Pooled)

$\mathrm{H}_{1}$ : Não há igualdade de interceptos e inclinações para toda a amostra. (Modelo com Efeitos Fixos)

Teste Cross-Section e período com efeitos fixos

Fonte: Dados da pesquisa (2019).

Para se determinar entre o Modelo com Efeitos Fixos (Fixed Effects) e o Modelo com Efeitos Aleatórios (Random Effects), aquele que melhor se ajusta aos dados da pesquisa, foi aplicado o Teste de Hausman. A significância estatística apresentada pelo $p$-value $<0,05(0,0036)$ levou à rejeição da hipótese nula que considera o Modelo com Efeitos Aleatórios (Random Effects) como aquele que oferece estimativas dos parâmetros mais consistentes para a variável dependente da pesquisa ROIC $_{\text {AJUSTADO, essa a proxy representativa }}$ da rentabilidade para os bancos. Os resultados obtidos com a aplicação do Teste de Hausman são apresentados na Tabela 7.

\section{Tabela 7}

Teste de Hausman - Regressão com a Variável Dependente ROICAJUSTADO

\begin{tabular}{|c|c|c|c|}
\hline Teste & Estatística Chi-quadrado & Graus de Liberdade & Probabilidade \\
\hline Cross-section aleatório & 0,000000 & 10 & 1,0000 \\
\hline Período aleatório & 0,000000 & 10 & 1,0000 \\
\hline Cross-section e período aleatórios & 26,127975 & 10 & 0,0036 \\
\hline \multicolumn{4}{|c|}{ Hipóteses para o Teste de Hausman ( $p$-value <0,05 rejeita-se a hipótese nula Ho) } \\
\hline \multicolumn{4}{|c|}{$\mathrm{H}_{0}$ : O Modelo Efeitos Aleatórios oferece estimativas dos parâmetros mais consistentes. (Efeitos Aleatórios) } \\
\hline \multicolumn{4}{|c|}{$\mathrm{H}_{1}$ : O Modelo Efeitos Aleatórios não oferece estimativas dos parâmetros mais consistentes. (Efeitos Fixos) } \\
\hline \multicolumn{4}{|c|}{ Teste Cross-Section e período com efeitos aleatórios } \\
\hline
\end{tabular}

Desse modo, é aceita a hipótese alternativa que considera o Modelo com Efeitos Fixos (Fixed Effects) como o mais adequado e consistente para os dados da pesquisa. Os resultados obtidos com a estimação da regressão com dados em painel no Modelo com Efeitos Fixos (Fixed Effects) estão apresentados na Tabela 8. As variáveis independentes consideradas estatisticamente significativas para os dados da pesquisa são aquelas que apresentaram como resultados para o p-value um nível de significância estatística de 1\% e de $5 \%$. 
A Estrutura de Capital é Relevante para a Rentabilidade dos Bancos? Evidências Empíricas nos Maiores Bancos Brasileiros com Papéis Negociados na [B]3

\section{Tabela 8}

Regressão com Dados em Painel no Modelo com Efeitos Fixos (Fixed Effects)

\begin{tabular}{|c|c|c|c|c|c|}
\hline Variável Independente & Coeficiente & Erro Padrão & Estatística t & Probabilidade & (a) \\
\hline (Constante) & 16,31566 & 1,854495 & 8,797901 & 0,0000 & \\
\hline CAP & 0,167734 & 0,055810 & 3,005429 & 0,0032 & $* * *$ \\
\hline LEV & 0,132290 & 0,055946 & 2,364601 & 0,0197 & ** \\
\hline CUS & $-0,186878$ & 0,010634 & $-17,57380$ & 0,0000 & $* * *$ \\
\hline SPR & 0,003577 & 0,004611 & 0,775751 & 0,4394 & \\
\hline CRE & $-0,032092$ & 0,027785 & $-1,155007$ & 0,2504 & \\
\hline ENC & $-0,025004$ & 0,307152 & $-0,081406$ & 0,9353 & \\
\hline LIQ & 0,029913 & 0,037578 & 0,796035 & 0,4276 & \\
\hline RIS & 0,112225 & 0,044942 & 2,497115 & 0,0139 & ** \\
\hline TES & 0,009588 & 0,022217 & 0,431574 & 0,6668 & \\
\hline $\mathrm{IMO}$ & $-0,013743$ & 0,005702 & $-2,410175$ & 0,0175 & ** \\
\hline $\mathrm{R}^{2}$ & 0,939918 & \multirow{4}{*}{\multicolumn{4}{|c|}{$\begin{array}{l}\text { (a) Variáveis independentes estatisticamente significativas ao nível } \\
\text { de: } * * * 1 \% ; * * 5 \% ; e^{*} 10 \% \text {. }\end{array}$}} \\
\hline R2 Ajustado & 0,911645 & & & & \\
\hline F-statistic & 33,24359 & & & & \\
\hline Prob. (F-statistic) & 0,000000 & & & & \\
\hline \multicolumn{6}{|c|}{ Variável Dependente: ROICAUUSTADo } \\
\hline \multicolumn{6}{|c|}{ Amostra: 2008Q1 até 2018Q4 - Períodos Incluídos: 44} \\
\hline \multicolumn{6}{|c|}{ Cross-Sections incluídas: 4 (BRAD, BRAS, ITAU e SANT) } \\
\hline \multicolumn{6}{|c|}{ Total de observações no Painel Balanceado: 176} \\
\hline
\end{tabular}

Os resultados fornecidos pelo Modelo com Efeitos Fixos (Fixed Effects) indicam que as variáveis CAP (Capitalização), LEV (Leverage), CUS (Custo Operacional), RIS (Risco de Crédito) e IMO (Grau de Imobilização) mostraram-se estatisticamente significativas para a proxy de rentabilidade ROICAJUSTADO, sendo, portanto, capazes de influenciar a rentabilidade dos bancos. Cabe destacar que os sinais obtidos nesses resultados se apresentam em conformidade com a relação esperada na Teoria de Finanças e nas pesquisas empíricas que analisaram a rentabilidade nos bancos.

Diante do exposto, permite-se então rejeitar a Hipótese Nula $\left(H_{0}\right)$ de que não há relação estatisticamente significativa entre a estrutura de capital e a rentabilidade dos bancos, sendo, portanto, aceita a Hipótese Alternativa $\left(\mathrm{H}_{1}\right)$ que declara existir relação estatisticamente significativa entre a estrutura de capital e a rentabilidade dessas empresas. Em outras palavras, poder-se-ia admitir a relevância da estrutura de capital para a rentabilidade dos bancos, dado que as variáveis independentes LEV (Leverage) e CAP (Capitalização) mostraram-se estatisticamente significativas ao nível de significância de $1 \%$ e de $5 \%$ na pesquisa.
A Hipótese Alternativa adicional $\left(\mathrm{H}_{1,1}\right)$ que considera a alavancagem financeira (leverage) relevante para a maximização de valor, influenciando positivamente a rentabilidade dos bancos, também foi aceita para a proxy de rentabilidade ROICAJUSTADO, ocasião essa em que LEV (Leverage) apresentou relação positiva e estatisticamente significativa ao nível de significância de 5\%. Com relação à Hipótese Alternativa adicional $\left(\mathrm{H}_{1,2}\right)$ que declara ser a CAP (Capitalização) dos bancos relevante para a maximização de valor, influenciando positivamente na rentabilidade, ela foi possível de ser aceita para a proxy de rentabilidade ROICAJUSTADO ao nível de significância de $1 \%$.

Ressalte-se que embora os resultados encontrados para LEV (Leverage) e CAP (Capitalização) atendam as hipóteses alternativas de pesquisa $\left(\mathrm{H}_{1,1}\right.$ e $\left.\mathrm{H}_{1,2}\right)$, tanto a Teoria de Finanças como as pesquisas empíricas desenvolvidas apontam para uma relação esperada ambígua entre essas variáveis e a rentabilidade dos bancos. Em outras palavras, LEV (Leverage) e CAP (Capitalização), como proxies representativas para a estrutura de capital, podem influenciar tanto de forma positiva, quanto de forma negativa, a rentabilidade dos bancos. 
A Estrutura de Capital é Relevante para a Rentabilidade dos Bancos? Evidências Empíricas nos Maiores Bancos Brasileiros com Papéis Negociados na [B]3

A Hipótese Alternativa adicional $\left(\mathrm{H}_{1,3}\right)$ que declara ser as estratégias bancárias relacionadas à eficiência operacional, solvência e liquidez capazes de influenciar a rentabilidade dos bancos, pode ser parcialmente aceita com base nos resultados obtidos para CUS (Custo Operacional), ao nível de significância estatística de 1\%, e para RIS (Risco de Crédito) e IMO (Grau de Imobilização), ao nível de significância estatística de 5\%. A relação apresentada por CUS (Custo Operacional) e por IMO (Grau de Imobilização) com a proxy de rentabilidade para os bancos ROICAJUSTADO foi negativa, enquanto RIS (Risco de Crédito) apresentou uma relação positiva. Os sinais obtidos por essas proxies estatisticamente significativas, as quais representam as estratégias bancárias relacionadas à eficiência operacional, estão em conformidade com a relação esperada na Teoria de Finanças e com as pesquisas empíricas que se dedicaram na análise da rentabilidade dos bancos.

\section{Conclusão}

A presente pesquisa, de natureza empíricoanalítica, descritiva, de abordagem quantitativa, teve por objetivo analisar a relevância da estrutura de capital para a rentabilidade dos quatro maiores bancos de capital aberto no Brasil. Além disso, verificou ainda se as principais estratégias bancárias relacionadas à eficiência operacional, solvência e liquidez são capazes de influenciar a rentabilidade dos bancos. O período contemplado nas análises considerou o intervalo entre 0 10 trimestre de 2008 até o 4 o trimestre de 2018. Um conjunto de dados de corte transversal com 4 bancos associados a uma série temporal de 44 trimestres, totalizando 176 observações, foi utilizado para a pesquisa.

Os resultados estatisticamente significativos obtidos para as variáveis CAP (Capitalização) e LEV (Leverage) indicam a relevância da estrutura de capital para a rentabilidade dos bancos. O impacto positivo da CAP (Capitalização) sobre a rentabilidade dos bancos, a qual foi mensurada pelo ROICAJUSTADO, pode ser justificada pelo fato das empresas bem capitalizadas serem capazes de reduzir os custos de financiamentos e de captação, ao sinalizarem ao mercado uma menor possibilidade de falências, estando assim sujeitas a menores custos de falências esperados (D'Oliveira, 2014).

Já a relação positiva apresentada por LEV (Leverage) com a rentabilidade dos bancos pode ser explicada pelo fato da elevada dependência de recursos de terceiros (leverage) na estrutura de capital possibilitar aos bancos uma alta capacidade de alavancagem de seus resultados (Assaf, 2015). Maiores níveis de depósitos de clientes compondo a estrutura de capital dessas empresas geram maior volume de recursos disponíveis para a intermediação financeira (Mendonça et al., 2016), representando uma forma de captação menos onerosa para o incremento da rentabilidade, tendo em vista que esse tipo de recurso pode ser assegurado por algum órgão privado ou governamental, como por exemplo, os seguros de depósitos que contribuem para a redução de riscos e de custos de captação perante os depositantes (Vieira, 2016). Além disso, o endividamento na estrutura de capital proporciona um beneficio fiscal pela dedutibilidade dos juros incorridos sobre - lucro tributável, servindo ainda como instrumento de disciplina para mitigar o conflito de interesses entre acionistas e administradores (Vieira, 2016).

A significância estatística e a influência negativa de CUS (Custo Operacional) sobre a rentabilidade dos bancos, mensurada pelo ROICAJUSTADO, pode ser explicada pelo fato dos custos operacionais serem considerados significativos nestas empresas, especialmente os elevados custos fixos incorridos para configuração da rede de agências e da infraestrutura de tecnologia da informação, bem como, os elevados custos variáveis decorrentes da própria operação (D'Oliveira, 2014). Os bancos que utilizam eficientemente seus recursos obtêm uma redução dos custos operacionais e um aumento no nível dos serviços, melhorando assim a sua eficiência operacional e seu desempenho econômico-financeiro, possibilitando agregar valor para a empresa e incrementar sua rentabilidade, gerando maiores retornos para acionistas e demais stakeholders (Souza \& Macedo, 2009; Mendonça et al., 2016; Vieira, 2016).

Outra informação obtida com base nos resultados da pesquisa diz respeito à significância estatística e relação negativa de IMO (Grau de 
Imobilização) com a rentabilidade dos bancos mensurada pelo ROICAJustado. O grau de imobilização indica a proporção do capital próprio dos bancos que está aplicado em ativos imobilizados, ou seja, ativos que essas empresas utilizam para prestação de serviços aos clientes e ao público em geral (Assaf, 2015). Os bancos que conseguem uma redução no nível de imobilização (imóveis, terrenos, veículos, entre outros), mantendo a atual estrutura para atendimento de um maior número de demandas de seus clientes, ou então, promovendo uma redução em seus custos, são capazes de obter melhorias em eficiência e rentabilidade (Souza \& Macedo, 2009; Bittencourt et al., 2017).

O RIS (Risco de Crédito) também se mostrou estatisticamente significativo e com relação positiva com a proxy de rentabilidade ROICAJUSTADO. Esse tipo de risco é o mais óbvio para os bancos, dado sua função de intermediador financeiro, podendo surgir quando os fluxos de caixa esperados para os títulos primários possuídos pelos bancos revelam indícios de probabilidade de liquidação duvidosa futura por parte de seus clientes (Saunders, 2000). Ainda que maiores níveis de provisões nesse sentido aumentem os riscos e os custos de captação e de oportunidade do dinheiro provisionado, elas podem revelar um reconhecimento oportuno de empréstimos sob liquidação duvidosa como parte de determinada estratégia adotada pelos bancos, afinal as maiores taxas que passam a ser exigidas sobre as operações de empréstimos podem terminar em maiores lucros para essas empresas (D'Oliveira, 2014). Assim, se por um lado os bancos devem realizar o reconhecimento de maiores montantes de provisões para cobrir perdas com operações de crédito mais arriscadas, por outro lado eles podem cobrar juros mais altos nessas operações, aumentando assim a sua lucratividade (Vieira, 2016).

Embora os sinais obtidos para os resultados das variáveis estatisticamente significativas estejam em conformidade com a relação esperada na Teoria de Finanças, eventuais desvios ou incompatibilidade de sinais podem decorrer das diferentes estratégias comercial e de crédito, de fusões e aquisições e de políticas de investimentos, financiamentos e de distribuição de dividendos praticadas pelos bancos que compõem a amostra da pesquisa. Ressalte-se que a inclusão de um banco público e de um banco privado de controle externo na amostra de pesquisa, além do cenário instável no período da análise, são alguns aspectos que podem ter contribuído para a exclusão de determinadas variáveis dos resultados estatisticamente significativos apresentados pela pesquisa.

Por fim, cabe mencionar que esta pesquisa não se propõe a esgotar o assunto, tampouco produzir conclusões definitivas acerca da questão de pesquisa explorada, sendo, portanto, sugerido para pesquisas futuras: (i) acrescentar novas variáveis e novo horizonte temporal nos estudos posteriores; (ii) confrontar o desempenho dos bancos desta pesquisa com os principais bancos dos EUA e de países emergentes e/ou integrantes do BRICS; e (iii) analisar se as exigências de capital regulatório previstas nos Acordos de Basileia contribuem, de forma estatisticamente significativa, para alterações na rentabilidade dos bancos.

\section{Referências}

Aboura, S., \& Lépinette, E. (2015). Do banks satisfy the Modigliani-Miller theorem? Economics Bulletin, 35(2), 924-935.

Andrade, C. R. (2014). Basileia III: Uma análise das novas recomendações do Comitê de Supervisão Bancária da Basileia sobre o setor financeiro brasileiro (Dissertação de Mestrado). Universidade Federal do Rio Grande do Sul, Porto Alegre, RS, Brasil.

Assaf, A., Neto. (2015). Estrutura e análise de balanços: Um enfoque econômico-financeiro (11a ed.). São Paulo: Atlas.

Banco Central do Brasil. (2017). Plano contábil das instituições do Sistema Financeiro Nacional (COSIF). Banco Central do Brasil (BACEN). Brasília: Autor.

Banco Central do Brasil. (2019). IF.data - Dados selecionados de instituições financeiras. Banco Central do Brasil (BACEN). Brasília: Autor. 
Belém, V. C., \& Gartner, I. R. (2016). Análise empírica dos buffers de capital dos bancos brasileiros no período de 2001 a 2011. Revista de Contabilidade e Finanças USP. São Paulo, 27(70), 113-124.

Berger, A. N., Herring, R. J., \& Szegö, G. P. (1995). The role of capital in financial institutions. Wharton Financial Institutions Center. (Working Paper n. 95-01), 1-55.

Bittencourt, W. R., Bressan, V. G. F., Goulart, C. P., Bressan, A. A., Costa, D. R. M., \& Lamounier, W. M. (2017). Rentabilidade em bancos múltiplos e cooperativas de crédito brasileiros. Revista de Administração Contemporânea. Rio de Janeiro, 21, 22-40.

Caliman, L., Sarlo Neto, A., Reina, D., \& Junior, E. M. (2016). Bancos Comerciais e Múltiplos versus Cooperativas de Crédito: Uma Análise Comparativa dos Indicadores Econômico-Financeiros. Anais do XIX Seminários em Administração - SemeAd, São Paulo, SP, Brasil.

Ceretta, P. S., \& Niederauer, C. A. P. (2001). Rentabilidade e Eficiência no Setor Bancário Brasileiro. Revista de Administração Contemporânea. Rio de Janeiro, 5(3), 7-26.

Chen, S. (2014). Integrated bank analysis and valuation: A practical guide to the ROIC methodology. The Palgrave Macmillan, London.

Coelho, E. G. (2015). Estrutura de Capital: Um estudo bibliométrico dos reflexos de seus determinantes. Anais do XV Congresso USP de Controladoria e Contabilidade 2015: Contabilidade e Controladoria no Século XXI. São Paulo, SP, Brasil.

Copeland, T., Koller, T., \& Murrin, J. (2002). Avaliação de empresas - valuation: Calculando e gerenciando o valor das empresas. (3a ed.). São Paulo: Pearson Brasil.

Creswell, J. W. (2010). Projeto de pesquisa: Métodos qualitativo, quantitativo e misto. (3a ed.). Porto Alegre: Artmed.
Diamond, D. W., \& Rajan, R. G. (2001). Liquidity risk, liquidity creation, and financial fragility: A theory of banking. Journal of Political Economy, 109(2), 287-327.

D'Oliveira, E. H. (2014). Determinantes da lucratividade bancária no Brasil (Dissertação de Mestrado). Universidade de Brasília - UnB, Brasília, DF, Brasil.

Duarte, P. C., Lamounier, W. M., \& Takamatsu, R. T. (2007). Modelos econométricos para dados em painel: Aspectos teóricos e exemplos de aplicação à pesquisa em contabilidade e finanças. Anais do IV Congresso USP de Iniciação Científica em Contabilidade 2007, São Paulo, SP, Brasil.

Gonçalves, R. S., Tavares, A. L., Ximenes, P. M., \& Silva, R. M. P. (2012). Comportamento dos dez maiores bancos brasileiros durante a crise do subprime: Uma análise por meio de indicadores contábeis. Revista de Educação e Pesquisa em Contabilidade. Brasília, 6(2), 170-186.

Gray, D. E. (2012). Pesquisa no mundo real. (2a ed.). Porto Alegre: Penso.

Greene, W. H. (2012). Econometric analysis. (7a ed.). International Edition: Pearson.

Gujarati, D. N., \& Porter, D. C. (2011). Econometria básica. (5a ed.). Porto Alegre: AMGH.

Hair, J. F., Jr., Babin, B., Money, A. H., \& Samouel, P. (2005). Fundamentos de métodos de pesquisa em administração. Porto Alegre: Bookman.

Jensen, M. C. (1986). Agency costs of free cash flow, corporate finance and takeovers. American Economic Review, 76(2), 323-329.

Jokipii, T., \& Milne, A. (2008). The cyclical behavior of European bank capital buffers. Journal of Banking and Finance, 32(8), 1440-1451.

Jucá, M. N. (2011). Determinantes da estrutura de capital de bancos brasileiros e norte-americanos 
(Tese de Doutorado). Universidade de São Paulo, São Paulo, SP, Brasil.

Maffili, D. W., Bressan, A. A., \& Souza, A. A. (2007). Estudo da rentabilidade dos bancos brasileiros de varejo no período de 1999 a 2005. Contabilidade Vista e Revista, 18(2), 117-138.

Mantovani, M. H. C.; Santos, J. O. (2015). Análise da Relação entre Alavancagem e Rentabilidade dos Bancos Brasileiros Listados na Bolsa de Valores de São Paulo no Período de 2001 a 2010. REGE Revista de Gestão, 22(4), 509-524.

Martin, D. M. L., Kimura, H., Kayo, E. K., \& Santos, F. H. L. (2011). Determinantes da Lucratividade de Grandes Bancos Brasileiros: Uma Abordagem em Painel com DEA e MPI. Anais do $X X X V$ Encontro da ANPAD - EnANPAD, Rio de Janeiro, RJ, Brasil.

Martins, G. A. (2002). Manual para elaboração de monografias e dissertações. (3a ed.). São Paulo: Atlas.

Mendonça, D. J., Souza, J. A., Benedicto, G. C., \& Silva, S. N. A. (2016). Relação entre eficiência econômico-financeira e lucratividade em instituições bancárias brasileiras. Anais do XIX Seminários em Administração - SemeAd, São Paulo, SP, Brasil.

Menezes, G. (2017). Dados de painel no EViews: Technical report. ResearchGate, 1-24.

Modigliani, F., \& Miller, M. H. (1958). The cost of capital, corporation finance and the theory of investment. The American Economic Review, 48(3), 261-297.

Octavia, M., \& Brown, R. (2008). Determinants of bank capital structure in developing countries: regulatory capital requirement versus the standard determinants of capital structure. (Working Paper). Social Science Research Network.

Oliveira, V. H., Macedo, M. A. S., \& Corrar, L. J. (2011). Estudo do Desempenho dos Maiores Bancos de Varejo no Brasil por meio da Análise
Envoltória de Dados (DEA). Revista de Informação Contábil - RIC, 5(2), 1-20.

Orgler, Y. E., \& Taggart, R. A., Jr. (1983). Implications of corporate capital structure theory for banking institutions. Journal of Money, Credit and Banking, 15(2), 212-221.

Pinheiro, F. A. P., Savóia, J, R. F., \& Securato, J. R. (2015). Basileia III: Impacto para os bancos no Brasil. Revista Contabilidade e Finanças, São Paulo, 26(69), 345-361.

Primo, U. R., Dantas, J. A., Medeiros, O. R., \& Capelletto, L. R. (2013). Determinantes da Rentabilidade Bancária no Brasil. BASE - Revista de Administração e Contabilidade da Unisinos, 10(4), 308-323.

Rime, B. (2001). Capital requirements and bank behavior: Empirical evidence for Switzerland. Journal of Banking and Finance, 25(4), 789-805.

Romdhane, M. (2010). The determinants of banks' capital ratio in developing countries: Empirical evidence from Tunisia. Social Science Research Network (SSRN).

Ross, S. A., Westerfield, R. W., Jaffe, J., \& Lamb, R. (2015). Administração financeira: versão brasileira de corporate finance. (10a ed.). Porto Alegre: AMGH Editora.

Rover, S., Tomazzia, E. C., \& Fávero, L. P. (2011). Determinantes econômico-financeiros e macroeconômicos da rentabilidade: Evidências empíricas do setor bancário brasileiro. Anais do XXXV Encontro da ANPAD - EnANPAD, Rio de Janeiro, RJ, Brasil.

Saunders, A. (2000). Administração de instituições financeiras: Tradução Antonio Zoratto Sanvicente. São Paulo: Atlas.

Souza, M. F. A., \& Macedo, M. A. S. (2009). Análise de desempenho contábil-financeiro no setor bancário brasileiro por meio da aplicação da análise envoltória de dados (DEA). BASE - Revista 
de Administração e Contabilidade da Unisinos, 6(2), 81-100.

Vallascas, F., \& Hagendorff, J. (2013). The risk sensitivity of capital requirements: evidence from an international sample of large banks. Review of Finance, 17(6), 1947-1988.

Vieira, C. A. M. (2016). Determinantes da lucratividade bancária: Evidências do sistema financeiro brasileiro. Anais do XIX Seminários em Administração - SemeAd, São Paulo, SP, Brasil.
Vinhado, F. S., \& Divino, J. A. (2013). Determinantes da rentabilidade das instituições financeiras no Brasil. Análise Econômica, 31(59), 77-101.

Wall, L. D., \& Peterson, P. P. (1998). The choice of capital instruments. Federal Reserve Bank of Atlanta, Economic Review, 4-17.

Wooldridge, J. M. (2002). Econometrics analysis of cross section and panel data. The MIT Press, Cambridge, MA.

\section{SOBRE OS AUTORES}

Alexandre Franco de Godoi - Pontifícia Universidade Católica de São Paulo - PUC, São Paulo, (Brasil). Email: godoi franco@hotmail.com. Orcid id: https://orcid.org/0000-0002-4879-8520

José Odálio dos Santos - Pontifícia Universidade Católica de São Paulo - PUC, São Paulo, (Brasil). Email: j.odalio@pucsp.br. Orcid id: https://orcid.org/0000-0001-6428-723X

Fernando de Almeida Santos - Pontifícia Universidade Católica de São Paulo - PUC, São Paulo, (Brasil). Email: almeidasantos@pucsp.br. Orcid id: https://orcid.org/0000-0002-1716-2802

José Carlos Marion - Pontifícia Universidade Católica de São Paulo - PUC, São Paulo, (Brasil). Email: jcmarion@usp.br. Orcid id: https://orcid.org/0000-0002-5641-6107 


\title{
DOES THE CAPITAL STRUCTURE RELEVANT TO BANKS' PROFITABILITY? EMPIRICAL EVIDENCE IN LARGEST BRAZILIAN BANKS WITH PAPERS TRADED IN [B] ${ }^{3}$
}

\author{
Alexandre Franco de Godoi, José Odálio dos Santos, Fernando de Almeida Santos \& José Carlos Marion \\ Pontifícia Universidade Católica de São Paulo - PUC, São Paulo, (Brasil)
}

\section{ARTICLE DETAILS}

\section{Article history:}

Received: 14 October 2019

Accepted: 28 September 2020

Available online January: 01 th 2021

Double Blind Review System

Scientific Editor

Ilan Avrichir

\section{Keywords}

Capital Structure Relevance Bank's Profitability Determinants Banking Performance

\section{ABSTRACT}

Objective: To identify evidence of the capital structure relevance and the main banking strategies for value maximization in banks.

Method: This is an empirical-analytical, descriptive research with a quantitative approach. Longitudinal data from the Economatica ${ }^{\circledR}$ database were collected from the first quarter of 2008 to the fourth quarter of 2018. Linear regression tests were performed for the analyzes (panel data).

Main results: There is evidence of the capital structure relevance and the influence of banking strategies on bank profitability measured based on ROIC ADJUSTED.

Relevance and originality: The timeliness, relevance and inevitable critical debates on the subject arouse the interest of the academic area for research directed to the critical analysis of the capital structure relevance and operational strategies for value maximization.

Theoretical Contributions: It provides the inevitable academic debate about capital structure relevance and key banking strategies for maximizing value in the largest publicly traded banks.

Social Contributions: Contributes to the decision-making process of sector managers, investors, financial analysts and regulatory bodies by addressing the capital structure relevance to banks, the risks and costs involved, the key aspects that encourage high levels of financial leverage and the determining factors for profitability. 


\title{
¿ES LA ESTRUCTURA DE CAPITAL RELEVANTE PARA LA RENTABILIDAD BANCARIA? EVIDENCIA EMPÍRICA EN LOS PRINCIPALES BANCOS BRASILEÑOS CON ACCIONES COTIZADAS EN [B] ${ }^{3}$
}

\author{
Alexandre Franco de Godoi, José Odálio dos Santos, Fernando de Almeida Santos \& José Carlos Marion \\ Pontifícia Universidade Católica de São Paulo - PUC, São Paulo, (Brasil)
}

\section{HISTORIA DEL ARTÍCULO}

Historia del Artículo:

Recibido: 14 de Octubre de 2019

Aceptado: 28 de septiembre de 2020

Disponible en línea: 01 de Enero 2021

Double Blind Review System

Editor Científico

Ilan Avrichir

Palabras-clave:

Relevancia de la Estructura de Capital Determinantes de la Rentabilidad

Bancaria

Desempeño de los Bancos

\section{RESUMEN}

Objetivo: Identificar evidencia de la relevancia de la estructura de capital y de las principales estrategias bancarias para la maximización del valor en los bancos.

Metodología: Se trata de una investigación empírica-analítica, descriptiva, con un enfoque cuantitativo. Los datos longitudinales se obtuvieron de la base de datos Economatica ${ }^{\circledR}$ entre el primer trimestre de 2008 y el cuarto trimestre de 2018. Se realizaron pruebas de regresión lineal con datos de panel (panel data).

Principales resultados: Existe evidencia de la relevancia de la estructura de capital y la influencia de estrategias bancarias para la rentabilidad de los bancos medida con base en ROICAJUSTADO.

Relevancia y originalidad: La actualidad, relevancia y la discusión crítica inevitable sobre el tema despiertan el interés del ámbito académico por la investigación dirigida al análisis crítico de la relevancia de la estructura de capital y las estrategias operativas para la maximización del valor.

Contribuciones teóricas: Proporciona el inevitable debate académico sobre la relevancia de la estructura de capital y las principales estrategias bancarias para la maximización del valor en los principales bancos brasileños cotizados en la bolsa.

Contribuciones sociales: Contribuye al processo de toma de decisiones de gestores, inversores, analistas financieros y organismos reguladores del sector al abordar la relevancia de la estructura de capital para los bancos, los riesgos y costos involucrados, los principales aspectos que incentivan niveles elevados de apalancamiento financiero y factores determinantes para la rentabilidad.

\section{Como citar este artigo:}

Godoi, A., Santos, J., Santos, F., \& Marion, J. (2021). A Estrutura de Capital é Relevante para a Rentabilidade dos Bancos? Evidências Empíricas nos Maiores Bancos Brasileiros com Papéis Negociados na [B]3. Internext, 16(1), 19-35. doi:http://dx.doi.org/10.18568/internext.v16i1.577 